\title{
ELABORATING THE MECHANISM OF CELL KILLING OF A NOVEL CHEMOTHERAPEUTIC DRUG TARGETING BREAST CANCER CELLS
}

Phat Do, Sridhar Varadarajan ${ }^{\mathrm{b}}$, Giridhar Akkaraju ${ }^{\mathrm{a}}$

aDepartment of Biology, Texas Christian University, Fort Worth, TX 76129, USA

${ }^{b}$ Department of Chemistry and Biochemistry, University of North Carolina Wilmington, Wilmington, NC 28403-5932, USA 


\section{INTRODUCTION}

Breast cancer $(\mathrm{BC})$ is the second most commonly diagnosed cancer in women in the world. In 2018, there were more than 2 million new diagnosis. It is estimated that 1 in 8 women will develop invasive breast cancer over the course of her lifetime. As such, the impact of breast cancer needs to be addressed. Traditional treatments of BC include surgery, radiation, and chemotherapy therapy; however, these treatments are non-specific and potentially kill peripheral, healthy cells. More specific treatments are needed, most notably to target a unique feature of the cancer cells. Interestingly, 70\% of BC cells upregulate estradiol-dependent pathway, a characteristic essential for rapid cell growth. Current BC drugs, such as Tamoxifen, Faslodex, or Femara have targeted this pathway to preferentially kill BC cells. However, the problems with these drugs are two-fold. (1) Drugs produce considerable side effects. For example. Femera causes considerable musculoskeletal failures. Tamoxifen is also shown to produce secondary cancer growth, such as endometrial cancer. (2) Breast cancer cells resist drugs very quickly. For instance, one third of women who are treated with Tamoxifen for five years relapse within fifteen years. The resulting tumor then become resistant to Tamoxifen treatment. For these two reasons, there is a need for new chemotherapeutic drugs.

Our research group studies a novel estrogen-receptor targeting drug: Estradiol-R-Melex. This compound has the estradiol molecule linked to a DNA alkylating agent, Melex. We connected the two moieties using a linker consisting of various lengths, i.e., one, two, and three methyl groups. The linker length variation is to optimize the cell killing property of our small drug molecule.

We hypothesize that Est-n-Melex enters the ER positive cancer cells more rapidly than ER- normal cells. The Estradiol moiety recognizes and binds to the inactive Estrogen Receptor alpha (ER- $\alpha)$. ER- $\alpha$ then dimerizes to form an active dimer complex, which then enters the nucleus and binds to Estrogen Response Elements on the DNA. This movement positions the Melex moiety to be in close proximity with the DNA and allows the transfer of a methyl group to the N-3 adenine on the DNA. This DNA methylation causes single-stranded DNA breaks (ssDNA). We hypothesize that when there are some ssDNA breaks, cells recruit DNA repair enzymes, poly ADP ribose polymerase (PARP), to repair the DNA damage. When there are too many ssDNA breaks, cells will send out signals and self-destroy via programmed cell death, or apoptosis (Figure 1). 


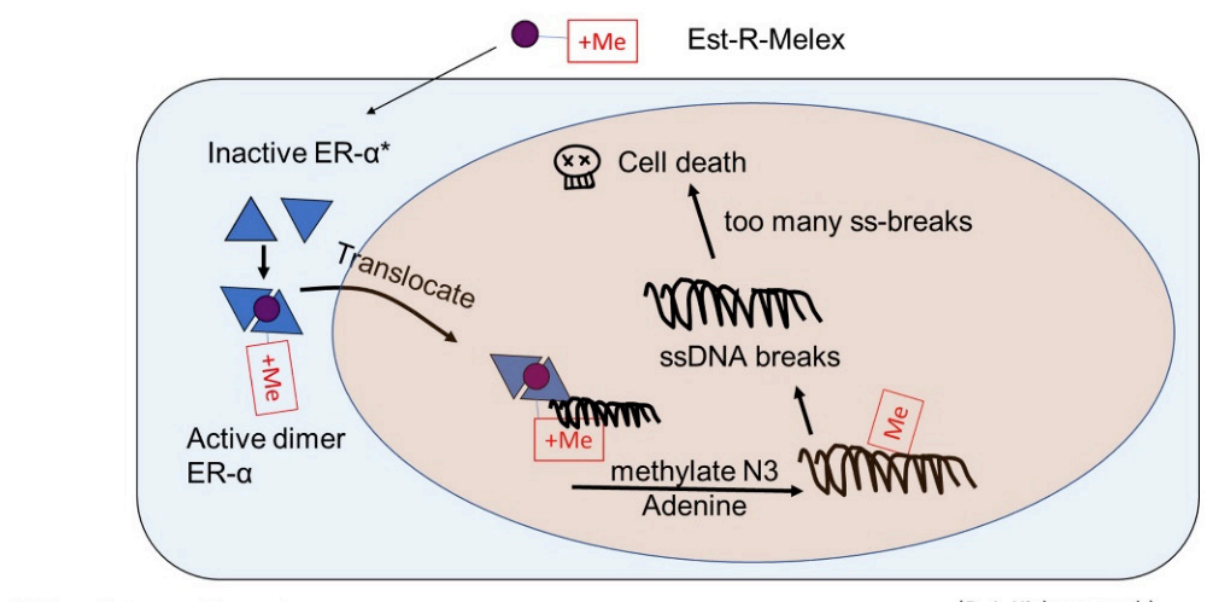

*ER-a: Estrogen Receptors $\alpha$

(R. J. Kishton et al.)

Figure 1 Hypothesized Mode of Action of Est-n-Melex in Estrogen Receptor positive cells.

\section{RESULTS AND DISCUSSION}

\section{Nuclear localization of small drug molecule}

The translocation of the Est-n-Melex was assessed in MCF-7 cells, a breast cancer cell line expressing the ER. Theoretical simulation modelling shows Est-n-Melex binds to the A/T rich minor groove of DNA. To confirm the model simulation, Hoechst-also an $A / T$ rich, minor-groove DNA binding agent, is used to compete with Est-n-Melex for the binding site. Since Est-3-Melex is cytotoxic and invisible under the microscope, its movement is tracked via a non-cytotoxic, florescent analogue, Est-3-NBD. The cells were exposed to a noncytotoxic concentration of Hoechst, followed by increasing concentration of Est3-NBD. The results are shown in Figure 2.

Without Hoechst treatment, Est-3-NBD is primarily in the nucleus. This confirms the Est-3-NBD is nuclear localized. Hoechst pretreatment overnight followed by 2.5 uM Est-3-NBD observes nuclear localization of Hoechst but cytosolic presence of Est-3-NBD. This infers Hoechst prevents nuclear localization of Est-3-NBD. Increasing Est-3-NBD concentration to $5.0 \mu \mathrm{M}$ observes more cytosolic presence of NBD, confirming that Hoechst and Est-3NBD competes for the minor groove of the DNA. 
A

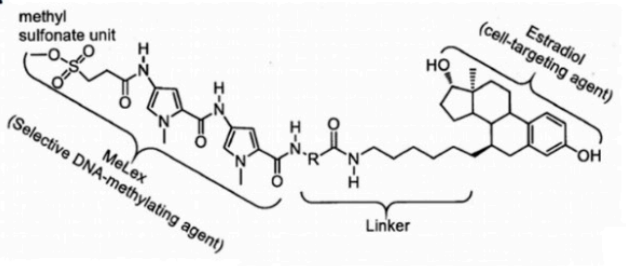

C

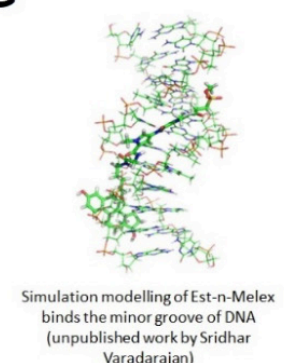
Varadarajant

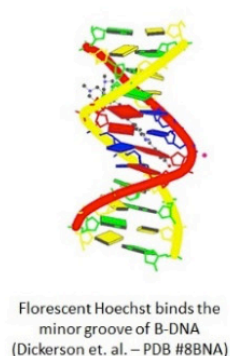
minor groove of B-DNA
(Dickerson et. al. - PDB \#8BNA)
B
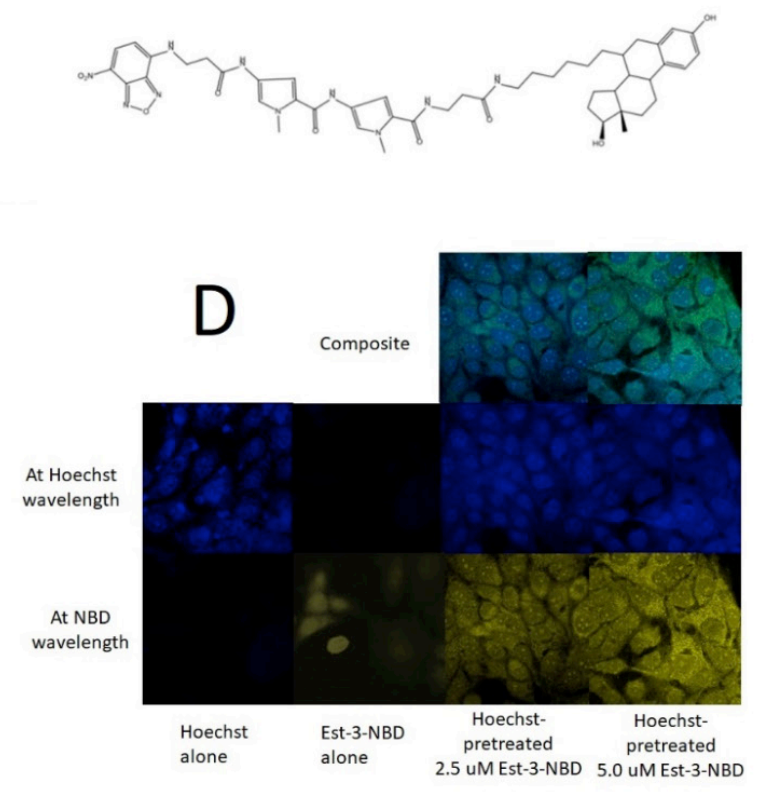

Figure 2 Est-n-NBD moves into the nucleus and binds to the minor groove of the DNA.

(A) Chemical structure of Est-3-Melex consisting of a DNA-alkylating agent conjugated to estradiol via a linker of 3 methyl groups. (B) Chemical structure of Est-3-NBD consisting of florescent NBD conjugated to estradiol via a linker of 3 methyl groups. (C) Model simulations of Est-n-Melex binding to the DNA vs. actual Hoechst binding to the DNA. (D) MCF-7 cells are pretreated with 6.05 e-5 mM of Hoechst overnight followed by increasing concentration of $2.5 \mu \mathrm{M}$ and $5.0 \mu \mathrm{M}$ of Est-3-NBD. Images are captured with confocal microscopy and composited with Image J.

Nuclear localization of target protein induced by Est-n-Melex

Immunofluorescence study is used to track ER- $\alpha$ localization upon Est$\mathrm{n}$-Melex treatment. Without the drug, ER- $\alpha$ remains cytosolic. Est-2-Melex treatment for 5 hours shows a more concentrated presence of ER- $\alpha$ nuclear localization of ER- $\alpha$. Prolonged exposure of the drug observes even a more concentration ER- $\alpha$ inside the nucleus (Figure 3). This shows that Est-2-Melex induces ER- $\alpha$ to localize into the nucleus. 


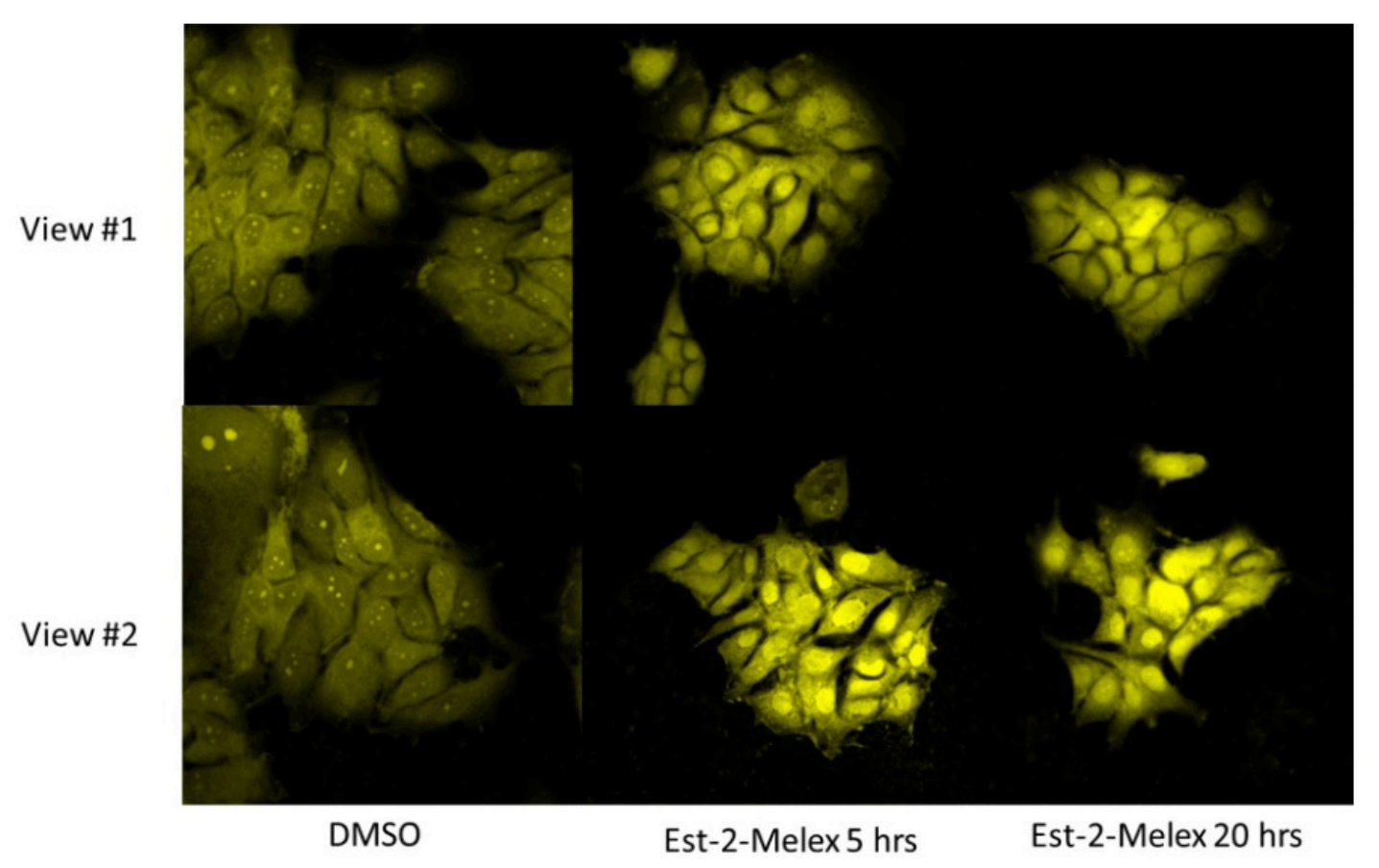

Figure 3 Est-2-melex induces nuclear translocation of ER- $\alpha$ in a time dependentmanner. 25,000 MCF-7 cells on cover-slip were treated with 7 uM estradiol (dissolved in Ethanol) or 75 nM est-2-melex (dissolved in DMSO) and let sit for $4 \mathrm{hrs}$ and $16 \mathrm{hrs}$. The samples were then treated with 7:500 a-ER antibody, followed by 1:1000 anti-mouse Alexafluro-488.

\section{SUMMARY}

In conclusion, the translocation mechanism of action of a novel chemotherapeutic compound that can target ER+ breast cancer cells has been confirmed. This compound targets $\mathrm{ER}+$ breast cancer cells and recognizes and binds to ER- $\alpha$, forming a complex that translocates into the nucleus. Experiments are currently in progress to elaborate further the cell killing mechanism of the drug, as well as the role of poly ADP ribose polymerase (PARP) - a DNA repair enzyme - in repairing DNA damage induced by Est-n-Melex. The successful elaboration of translocation mechanism of this compound demonstrates that this strategy can be used to synthesize new drugs that can preferentially target any cell-type that over-expresses a unique receptor or enzyme.

\section{ACKNOWLEDGMENTS}

Support of this research through grants from the Science and Engineering Research Center SERC of Texas Christian University is gratefully acknowledged. 


\section{REFERENCES}

Engelward, B. P.; Allan, J. M.; Dreslin, A. J.; Kelly, J. D.; Wu, M. M.; Gold, B.; Samson, L. D. J. Biol. Chem. 1998, 273, 5412. https://doi.org/10.1074/jbc.273.9.5412

Fronza, G.; Gold, B. J. Cell. Biochemistry 2004, 91, 250. https://doi.org/10.1002/ jcb.10698

Kishton, RJ; Miller, S. E.; Perry E.; Lynch T.; Patel M.; Gore V. K.; Akkaraju, G. R.; and Varadarajan, S. Bioorg Med Chem. 2011, 19, 17. https://doi.org/10.1016/j. bmc.2011.07.026

Zhang, Y.; Chen, F. X.; Mehta, P.; Gold, B. Biochemistry 1993, 32, 7954. https://doi. org/10.1021/bi00082a017 